\title{
L'initiation au Développement International en Afrique: Entre Mimétismes et Enjeux
}

\section{Honoré Gbedan,}

Doctorant en Sociologie, Université Laval (Québec, QC, Canada)

Doi:10.19044/esj.2019.v15n31p155 URL:http://dx.doi.org/10.19044/esj.2019.v15n31p155

\section{Résumé}

L'initiation au Développement international en Afrique présente d'importants enjeux souvent banalisés ou peu questionnés par la plupart des volontaires internationaux. Cet article se propose, à partir d'un cas pratique, d'analyser ces enjeux depuis l'installation du volontaire international dans sa famille ou communauté d'accueil jusqu'à la fin de son mandat. Il traite de ces enjeux en contexte de pratiques du développement, en se focalisant sur les acteurs, non pas ceux institutionnels, organisationnels et étatiques, mais plutôt sur des acteurs-individus. L'observation participante, l'observation directe et l'entretien indirect ont été les principaux instruments de collecte de données qui révèlent d'importants enjeux dont ceux du lien social, d'intégration, d'identification, du don, du contre-don et de pouvoir.

Mots-clés: Développement International, Solidarité Internationale, Enjeux, Don, Lien social, Mimétisme, Afrique subsaharienne

\section{Initiation to International Development in Africa: Between Mimicry and Issues}

\author{
Honore Gbedan, \\ Doctorant en Sociologie, Université Laval (Québec, QC, Canada)
}

\begin{abstract}
Initiation to International Development in Africa presents important issues that are often trivialized or not questioned by most international volunteers. Based on a studied case, this article aims at analyzing theses issues since the arrival of the international volunteer to his/her family or host
\end{abstract}


community until the end of his/her mandate. It analyzes these issues in the context of development practices, focusing on actors, not institutional, organizational and state actors, but rather on individual actors. Participating observation, direct observation and indirect interviewing are the main data collection tools that reveal important issues including social link, integration, identification, giving, counter giving and power.

Keywords: International Development, Global solidarity, Development issues, Giving, Social link, Mimicry, Sub-Saharan Africa

\section{Introduction}

Le monde du Développement International, cette croyance occidentale (Rist, 2013), a ses principes de fonctionnement dont la connaissance et/ou la maîtrise passeraient, soit par un premier $\operatorname{pas}^{10}$ (une sorte d'initiation), soit par un processus d'apprentissage, de formation; ou dans le meilleur des cas, les deux à la fois. Le propos développé dans cet article concerne un programme d'initiation au Développement International dans le cadre d'un projet de solidarité internationale au Sénégal, un des pays de l'Afrique Occidentale Française (AOF). Il s'agissait de mon séjour (été 2016) en tant que stagiaire bénévole international d'un projet d'agriculture biologique pour le développement durable au profit d'un groupement de femmes de Gapakh, un village de ce pays-là. Ledit stage bénévole a été une sorte de tremplin et de découverte du champ du développement international par la pratique et non une connaissance livresque de travaux topiques ou la participation à un cours au sens d'enseignement théorique. Précisément, le cas pratique qui a constitué la porte d'entrée sur cette arène (Olivier de Sardan, 1995) qu'est le développement international, est un projet financé par le Gouvernement du Québec, via une Organisation Non Gouvernementale (ONG) canadienne (au Québec) de solidarité internationale et au nom de laquelle les autres membres de l'équipe et moi avions reçu mandats.

Ledit projet n'était qu'une composante des activités de développement à la base que réalise une Organisation Non Gouvernementale (ONG) féminine locale partenaire de l'organisation internationale ${ }^{11}$ non gouvernementale canadienne (du Québec). En ce sens, l'organisation non gouvernementale sénégalaise avait agi en tant qu'intermédiaire entre l'organisation internationale canadienne et les populations bénéficiaires directes (le

\footnotetext{
${ }^{10} \mathrm{Je}$ voudrais remercier toute mon équipe de stage QSF.

${ }^{11}$ Bonnafous-Boucher et Dahl Rendtorff (2014, p. 62) définissent comme ONG internationales « ...des organisations d'intérêt public qui ne relèvent ni d'un État ni d'une institution internationale, des associations de droit privé et, en tant que telles, des personnes morales dont l'activité est internationale. »
} 
groupement de femmes du village) dudit projet. Autrement dit, cette ONG sénégalaise est un courtier du développement (Olivier de Sardan, 1995).

Dès lors, au-delà de la création des liens sociaux entre bénévoles internationaux (que nous étions), l'ONG locale intermédiaire, les populations bénéficiaires directes (les membres du groupement féminin) du projet et les communautés d'accueil d'une part et, de l'apprentissage du travail en équipe et du vivre en communauté ou de la découverte d'autres réalités et cultures (Binette, 2013) de l'autre, le programme d'initiation à la Solidarité internationale qu'est Québec Sans Frontières (QSF) présente d'importants enjeux a priori anodins, mais qui sont l'expression de réalités courantes du monde du Développement International. C'est de l'analyse de certains de ces enjeux qu'il s'agit dans le présent article. Cet article ne se propose donc pas de procéder à un état de l'art sur les enjeux ${ }^{12}$ du Développement international ou en Solidarité internationale. Le présent article traite des enjeux en contexte de pratiques du développement, en se focalisant sur les acteurs, non pas ceux institutionnels, organisationnels et étatiques, mais des individus. Il vise à principalement répondre à la double question de départ à savoir : quels sont les enjeux auxquels donne lieu un programme d'initiation à la Solidarité internationale en contexte socio-culturel sénégalais ? Et quelles stratégies les participant(e)s bénévoles à ce programme développent-ils pour une meilleure «première » expérience de terrain ?

Pour ce faire et compte tenu des réalités du terrain de l'étude, l'observation participante (Copans, 2008), l'observation directe (Arborio et Fournier, 2010), l'entretien compréhensif (Kaufmann, 2007) ${ }^{13}$,(sous forme de relances de questions suite à une intervention de femmes du groupement) non programmé et la tenue du journal de terrain ont été des instruments exploités dans mes collectes de données, dès notre arrivée dans la communauté villageoise sénégalaise qui nous avait réservé un accueil des plus chaleureux. L'analyse propositionnelle du discours (Bardin 1993, p. 244) a été, en grande partie, appliquée au matériau discursif obtenu. Le terrain, ce terme «... emprunté à l'anthropologie pour désigner ce qui est à la fois objet d'étude et lieu de séjour »(Combessie 1996, p. 15) est un village du Sénégal appelé Gapakh; notre village d'accueil.

${ }^{12}$ Le lecteur intéressé par cet aspect peut lire, entre autres travaux, ceux de : Baudet, P. et al. (2008). Introduction au développement international : approches, acteurs et enjeux, Ottawa : Presses de l'Université d'Ottawa. ; Beaudet, P. et Haslam, P.A. (dirs.). (2014). Enjeux et défis $d u$ développement international, Ottawa : Presses de l'Université d'Ottawa; Sequera Carvalho, J.A. (2003). Enjeux géopolitiques et nouvelles approches pour la coopération au développement, Paris : L'Harmattan.

${ }^{13}$ Kaufmann (2007, p. 26) « La démarche compréhensive s'appuie sur la conviction que les hommes ne sont pas de simples agents porteurs de structures mais des producteurs actifs du social, donc des dépositaires d'un savoir important qu'il s'agit de saisir de l'intérieur, par le biais du système de valeurs des individus ; ...» 
Le présent article est structuré en trois principaux points. En effet, le propos développé ici prend en compte la Solidarité internationale et l'installation des volontaires dans la communauté d'accueil, les enjeux du lien social, et les enjeux autour du don en solidarité internationale.

I- Solidarité internationale et installation des volontaires dans la communauté d'accueil

A- S'impliquer en solidarité internationale : quelles finalités ?

La solidarité internationale est la sphère du Développement International dont les principaux acteurs sont les organisations à but non lucratif issues de la société civile ${ }^{14}$. Leurs modes de fonctionnement rompent, en principe, les amarres avec l'État et le marché. C'est dans cette perspective que s'inscrit le propos développé dans cet article.

En effet, Québec Sans Frontières (QSF) est le programme du Gouvernement Québécois d'initiation à la solidarité internationale, auquel nous avions participé comme bénévoles internationaux, dans un village du Sénégal en 2016. Le programme QSF date de $1995^{15}$. Dans le cas d'espèce, il s'agissait d'un projet non moins pertinent pour les communautés d'accueil en général, les cibles primaires (un groupement de femmes), en particulier. Succinctement, ledit projet d'agriculture biologique s'inscrivait dans une perspective de développement durable. Un tel projet de sécurité alimentaire mis en œuvre à Gapakh, un village Sérères ${ }^{16}$ du Sénégal, était pour ma part, pertinent en ce qu'il constituait une alternative, à petite échelle soit-elle, au rouleau compresseur qu'est l'agriculture chimique à grande échelle. À ce niveau, c'est un truisme de dire que les effets de l'agriculture chimique sur l'écologie et l'environnement demeurent problématiques. Spécifiquement, l'agriculture chimique, prise dans son volet monoculture intensive avec l'emblavement de grands espaces cultivables, pose de sérieux problèmes vitaux voire existentiels aux populations pauvres, fondamentalement paysannes de l'Afrique subsaharienne. En dehors de ces aspects, le projet d'agriculture biologique pour le développement durable dans l'esprit de la solidarité internationale, soulève d'autres problématiques dont l'autonomisation des femmes, la question de l'accès à la terre de ces femmes et la sécurité alimentaire dans ledit village.

Nombreuses sont les raisons qui expliquent la participation active aux programmes/projets de Développement International (solidarité

\footnotetext{
${ }^{14}$ Armony (2008, p. 206), a présenté les différentes manières de saisir la société civile comme acteur du développement. En effet, la société civile peut être, selon les contextes, acteur local, acteur authentique, acteur non politique, acteur social, acteur légitime, acteur global.

${ }^{15}$ Mais la présence du Québec en Afrique ne date pas de 1995; elle date plutôt de 1959 avec l'envoi des premiers missionnaires Québécois du retour de la Chine (Beaudet, 2009, pp. 4143). Le Canada, quant à lui, y est présent depuis 1964 (Brown, 2008, p. 268).

${ }^{16}$ Les Sérères sont un groupe sociolinguistique au Sénégal.
} 
internationale). En effet, cela peut être pour « ...comprendre une autre réalité, s'initier à des enjeux internationaux, participer à une expérience collective, faire du bénévolat, vivre dans un contexte culturel différent, découvrir un autre pays...le passage de la théorie à la pratique... » (Binette, 2013, p. 214).

En plus de ces raisons énumérées par Binette (2013), la participation et l'implication de bénévoles internationaux dans des projets de Développement en Afrique subsaharienne par exemple, trouvent leurs justifications dans ce que Fassin (2010) nomme des sentiments moraux, c'està-dire, ces émotions qui nous portent vers les malheurs des autres et nous poussent à les corriger. Boltanski (2007) parlera de souffrance à distance. À cet effet, l'on pourrait aisément conjecturer que certaines images (des personnes de la race dite noire, pour la plupart du temps) déployées par des médias et réseaux sociaux, sont de nature à inciter des âmes sensibles à s'engager dans la solidarité internationale.

La durée de ce bénévolat international dans le compte du projet supra précisé, était de 75 jours, marqués par d'importants enjeux, a priori anodins.

B- Accueil et installation des volontaires internationaux : théâtre et danse folklorique comme moyens d'expression et d'enjeux

Après une pause d'un jour à Dakar, la capitale sénégalaise, nous voilà dans le village de mise en œuvre du projet, le samedi 04 juin 2016 . Une foule impressionnante nous y attendait déjà avec impatience, sous un arbre tenant lieu de place publique.

Il est ici important de s'appesantir un tant soit peu sur la description de l'accueil qui nous avait été réservé par la communauté villageoise.

Dans l'allocution de bienvenue du Chef du village, la séquence suivante est pleine d'implication : «Vous êtes là pour renforcer et non pour remplacer le groupement ... Je suis content du fait que cette opportunité ait réuni les deux villages séparés par le nouveau découpage administratif. » Après le chef village, c'était le tour d'une responsable de l'ONG intermédiaire (celle-ci est incontestablement rodée dans le discours du Développement international) et de la présidente du groupement des femmes dudit village, bénéficiaire direct du projet.

Ensuite, la danse folklorique dont le refrain en Wolof, se résumait en «Na Gor, Goré » ${ }^{17}$. En effet, un jeune homme, dans sa danse, courut, sortit du cercle, monta à moitié sur un arbre, descendit et termina la cadence sur les jambes d'une femme portant un bébé au dos, sous les cris mélangés de rires et

${ }^{17}$ Ce refrain que j'ai écrit au son a quelque chose d'amusant. En effet, selon l'explication qui m'a été donnée, Gor en Wolof signifie « Homme ». En résumé, la chanson d'accueil signifiait, au sens folklorique : c'est une affaire de gentleman. 
d'étonnements des toubabs ${ }^{18}$; puis rejoignit le cercle. Au même moment, très entreprenante, à peine dans la vingtaine d'années et vêtue d'une longue robe rose avec des brillantines, robe visiblement apprêtée pour la circonstance, une jeune fille n'hésita pas à complimenter le toubab qui était plus près de moi, en lui disant « Tu es belle » au lieu de « Tu es beau ! ». Au même moment, trois principaux joueurs de tam-tam se changèrent en tee-shirt blanc avec le logo d'Amnesty International. En haut, du côté droit de leur poitrine, la couleur (rouge) du message m'avait laissé pantois ${ }^{19}$ : HALTE AU MARIAGE FORCÉ ET PRÉCOCE. Alors, deux des femmes qui dansaient, soulevèrent le pan de leurs pagnes en dévoilant l'attrait de leurs jambes. Quelques jeunes et belles filles vinrent donner du sang neuf à la cohorte de ces mères de famille. La fin du théâtre sur le mariage forcé fut marquée par la danse d'une jeune femme dont la dépigmentation de la peau était avancée. Et la foule se disloqua aussitôt que les responsables de l'ONG locale partenaire se levèrent pour aller installer le deuxième groupe de stagiaires/bénévoles dans le village de N'Dinguiray, un village voisin.

Quatre points a priori anodins de cette brève description de notre accueil et installation dans notre village (Gapakh) méritent quelques commentaires. Il s'agit du discours du Chef du village, du tee-shirt d'Amnesty International, de la jeune fille dans la vingtaine et de la dernière femme qui avait dansé.

Le discours du chef du village était centré sur les enjeux du pouvoir. Dans ce sens, il ne serait pas exagéré de procéder modestement à son exégèse ${ }^{20}$. En effet, le chef du village était clair dans ce qu'il disait sous forme d'avertissement : vous les stagiaires Toubabs, c'est moi qui dirige ce village. Vous devez savoir que vous y êtes pour une activité ponctuelle. Pour cela, vous devez connaître votre limite et ne jamais franchir le rubicond. Ne vous substituez pas au groupement de femmes bénéficiaires directe du projet. Bref, nous sommes dans notre village. Concernant l'autre volet de la séquence de son discours rapportée, il n'est pas hors de propos de supposer qu'il y eût eu un différend entre son village (le village de Gapakh, notre communauté d'accueil) et l'autre (N'Dinguiray) lors du nouveau découpage administratif ou ledit village était sous son contrôle. Il aurait donc perdu des portions de son pouvoir. En toute évidence, le projet «Agriculture biologique pour un

${ }^{18}$ Les Blancs y sont appelés Toubabs. Cela n'a rien de péjoratif. La communauté d'accueil m'appelait toubab-bougnoule, c'est-à-dire, Blanc à la peau noire.

${ }^{19}$ Je ne voyais pas le lien direct entre ce message et le projet d'agriculture biologique pour lequel nous étions là.

${ }^{20} \mathrm{D}$ 'aucuns pourraient penser que ma tentative d'exégèse s'apparente à un procès d'intention. Ce qui n'est pas du tout le cas. J'étais présent lors de l'allocution de ce chef du village et mon interprétation est soutenue par un grand effort d'objectivité. 
développement durable » fut un élément de communion, de rapprochement, de renforcement de liens et donc de paix entre le village (Gapakh) où notre équipe avait travaillé et le village voisin (N'Dinguiray).

Le fait que les stagiaires fussent accueillis par un théâtre sur le mariage forcé qui n'avait aucun rapport avec le projet en Agriculture pour lequel nous étions là et que les danseurs eussent porté des tee-shirts d'Amnesty International (et non celui de l'ONG canadienne dont nous avions mandat), pose un problème de conflits d'intérêts. Cela était-il anodin ? Était-ce une bonne chose ? Au lecteur de l'apprécier. Ce que l'on puisse affirmer est qu'une telle pratique s'apparenterait au «détournement» d'activités de Développement. L'on pourrait cependant faire une conjecture qui mitigerait une telle pratique de l'ONG locale partenaire. L'on peut penser qu'il y avait possiblement conflit d'agenda d'une part et que dans ce village (Gapakh), il est a priori, difficile de mobiliser du monde. Toutes choses qui auraient conduit 1'ONG locale partenaire à décider de faire d'une pierre, deux coups.

La solidarité internationale, c'est aussi le mimétisme ${ }^{21}$, la « chasse » à l'âme sœur. Dans le cas d'espèce, le mimétisme était dermique. C'est-à-dire, la propension à utiliser de crèmes chimiques éclaircissant la peau afin qu'elle devienne comme celle des Blancs, même si la personne vit dans un pays où il $\mathrm{y}$ a le soleil pratiquement tous les jours et où la température ambiante est généralement à $37^{0} \mathrm{C}$. Le fait que la jeune femme sénégalaise à peau dépigmentée finît notre accueil par sa danse ne me paraissait pas anodin. En fait, l'on se souvient généralement des dernières images. De plus, ce ne serait pas exagéré de penser que le compliment de la jeune d'une vingtaine d'années à l'endroit du stagiaire près de moi, n'était pas anodin. Il y a donc d'enjeux de liens sociaux.

II- Les enjeux du lien social

A- $\quad$ Légitimation et estime sociale de la famille d'accueil

Il y a incontestablement un lien social qui se crée entre la famille d'accueil et le volontaire international. En effet, pour Cusset (2011, p. 5), le lien social « ...désigne de fait des réalités multiples, qui vont de l'ensemble des relations concrètes que l'on entretient avec sa famille, ses amis, ses collègues ou ses voisins, jusqu'aux mécanismes collectifs de solidarité, en passant par les normes, les règles, les valeurs et les identités qui nous dotent d'un minimum de sens d'appartenance collective. »

Précisément et dans le meilleur des cas, «Durant un stage QSF, on habite dans une communauté durant deux mois. On tisse des liens fraternels, ce qui crée un sentiment d'appartenance à cette deuxième famille étrangère. On s'attache, on développe des amitiés qui dureront » (Binette, 2013, p. 215). Dans le pire

${ }^{21}$ Je m'attarderai un tant soit peu sur le mimétisme au niveau des enjeux d'intégration et d'identification. 
des cas, le volontaire international QSF peut changer de famille d'accueil ou démissionner du programme.

Dans un village, héberger un ${ }^{22}$ volontaire international présente d'importants enjeux. En effet, la famille d'accueil du volontaire international est perçue, dans sa communauté, comme riche, saine, lettrée privilégiée et ouverte d'esprit. Cela donne plus de crédit, d'estime au sein de la communauté. C'est un facteur de légitimation et d'influence dans la communauté. Toutes choses susceptibles de nourrir la compétition entre des familles dans la communauté, quant aux possibilités d'héberger des volontaires internationaux futurs.

J'avais assisté, à distance, à une sorte de dispute lorsqu'une des responsables de l'ONG locale partenaire prévenait une famille qu'elle allait lui retirer le privilège d'héberger un volontaire international l'année suivante. L'on ne saurait, à ce niveau, exclure le risque de chantage, de manipulation et les abus. Le volontaire international, quant à lui, a des efforts d'intégration à faire.

B- Le dilemme de l'intégration du volontaire international : mimétisme ou identification?

L'intégration $\mathrm{du}$ volontaire international dans sa famille ou communauté d'accueil nécessite avant tout, le don de soi et le don du temps. En effet, le bénévole du programme Québec Sans Frontières (QSF), en plus des tâches qui lui sont confiées dans le cadre de son mandat, est appelé à intégrer sa famille d'accueil. Dès lors, toute attitude d'isolement volontaire de soi est mal perçue par non seulement la famille d'accueil, mais aussi par la communauté. Autrement dit, dans le contexte africain en général, celui du Sénégal spécifiquement, les rapports sociaux ou si l'on préfère, la nature des liens sociaux est très souvent déterminante dans la réussite des activités quotidiennes, celles du Développement International en particulier. Le volontaire QSF doit y attacher du prix. Pour ce faire, le volontaire international doit avoir l'intelligence et l'humilité aux fins du don de soi d'une part, et du don de temps, de l'autre. Le don de soi, le don de relation ne doit pas être pris au sens oblatif. Noël (2015, p.132-133) a d'ailleurs bien saisi cette réalité lorsqu'il écrit :

Dans ces cultures, la relation personnelle prime sur le contenu de l'affaire à régler. Il faut d'abord bien se connaître si on veut faire des affaires ensemble. Et cette prise de connaissance passe par la parole, bien sûr, mais aussi par le temps que l'on prend à l'établir. C'est en parlant ensemble, d'abord de tout et de rien, en sirotant quelques verres de thé, que peu à peu l'on s'apprivoise, que l'on en vient à se connaître, à comprendre quelles sont les valeurs des

${ }^{22} \mathrm{La}$ forme neutre est utilisée afin d'alléger la lecture de l'article. 
protagonistes, leurs réseaux de relations, et, petit à petit, au besoin par l'échange de quelques cadeaux, dont la valeur est secondaire par rapport au choix qui en est fait en fonction des goûts du destinataire, on en vient à parler de tout ce qui fait nos préoccupations communes.

Le don de relation, c'est la disposition du volontaire international à montrer son altruisme dans la consolidation du lien social créé entre lui et sa famille d'accueil ou la communauté. Le don du temps, c'est l'importance que ce dernier accorde à sa famille d'accueil et à la communauté par la socialisation. Dans ces perspectives, le volontaire international peut, par exemple, se faire confectionner des vêtements locaux qu'il porte au besoin ou selon des événements dans la famille d'accueil ou la communauté. C'est aussi et souvent le moment où il apprend la langue de sa famille d'accueil ou de la communauté ; s'habitue à leur alimentation ; cherche à connaître l'histoire de sa famille d'accueil et/ou de la communauté.

Mais ces efforts d'intégration du volontaire international peuvent vite se muer en mimétisme. En effet, il avait été constaté jusqu'à quel point certains « collègues » volontaires étaient à la limite du snobisme. Ce qui n'était pas le cas du côté de la famille ou la communauté d'accueil.

De façon générale, une culture hybride temporaire s'était développée ; un consensus tacite se dégageait et permettait la coexistence pragmatique des deux cultures différentes, ici, celle canadienne (Québec) et celle sénégalaise. Ces femmes sénégalaises bénéficiaires directes vivaient dans un état d'ingéniosité perpétuelle qui facilitait le brassage et l'hybridation culturels lors de la mise en œuvre dudit projet d'agriculture biologique de solidarité internationale, à travers entre autres choses, le don.

III- Les enjeux autour du don en solidarité internationale

A- Le don : la pierre angulaire de la solidarité internationale

La solidarité internationale est largement dominée par les Organisations de la Société Civile (OSC) parmi lesquelles les ONG tiennent le haut du pavé. De par leur principe de fonctionnement, celui de n'exercer principalement pas d'activité génératrice de revenu, ces organisations qui forment le tiers secteur, recourent le plus souvent au don philanthropique. Elles se distinguent donc du fonctionnement de l'État et du marché. Dans ce sens, Godbout (2000b, p. 12) précise que «Le marché est dominé par le principe de l'équivalence et la recherche de l'utilité (ou du profit) dans l'échange ; l'État est dominé par le principe de l'autorité et du droit et la recherche de l'égalité et de la justice ; la sphère des réseaux est dominée par le principe du don et de la dette. Cette dernière comprend l'univers des 
rapports personnels et celui des associations où domine le don entre étranger. $»^{23}$

Le don de la solidarité internationale est spécifique par nature. Il diffère du don ${ }^{24}$ spirituel, naturel ou inné. Ce n'est pas non plus le don rituel ou cérémoniel analysé par Mauss (2007). Il s'agit, comme l'a précisé l'anthropologue Hénaff (2002, p.55), du don moderne, non dans ses expressions individuelles qui résident dans le cadeau, mais dans celles institutionnelles matérialisées par l'aide humanitaire. C'est dans ce sens que le don est « ... toute prestation de biens ou de services effectuée, sans garantie de retour, en vue de créer, entretenir ou régénérer le lien social. » (Caillé 2007, p.124; Godbout 2000a, p.32). C'est également, comme le définit Godelier (2002, p.2), « ...un acte volontaire, individuel ou collectif, qui peut ou non avoir été sollicité par celui, celles ou ceux qui le reçoivent. » Un don appel généralement un contre-don.

B- Le contre-don en solidarité internationale

Le don de la solidarité internationale doit-il être un don gratuit ? Est-il un don gratuit (Nestor, 2016) au sens d'absence absolue de « retour»? Ou remplit-il la triple obligation : l'obligation de donner, l'obligation de recevoir et l'obligation de rendre au sens de Mauss dans son Essai (2007) ? Autrement dit, pourrait-on parler ici de la loi du $d o^{25}$ ?

Face à ces interrogations, une réalité en termes de pouvoir et de liens, demeure. Celle décrite par Godelier (2002, p.21) et qui mérite d'être soulignée. Selon ce sociologue,

Donner semble instituer simultanément un double rapport entre celui qui donne et celui qui reçoit. Un rapport de solidarité, puisque celui qui donne partage ce qu'il a, voire ce qu'il est, avec celui à qui il donne, et un rapport de supériorité, puisque celui qui reçoit le don et l'accepte se met en dette vis-à-vis de celui qui lui a donné. Par cette dette il devient son obligé, et donc se retrouve jusqu'à un certain point sous sa dépendance, du moins pour tout le temps où il n'aura pas « rendu » ce qu'on lui a donné.

C'est un don qui présente une sorte de paradoxe en ce sens que les populations bénéficiaires directes de ce don se voient souvent obligées de faire un « contredon » qui n'est pourtant pas exigé par le(s) donateur(s). À ce niveau du don de

\footnotetext{
${ }^{23}$ L'auteur reconnaît cependant que tous les principes sont présents dans tous ces trois secteurs cités, mais qu'ils y jouent un rôle différent et que leur articulation y diffère.

${ }^{24}$ En ce sens, nous disons avec Athané (2011, p.7) que le don dont il est question ici n'est pas celui analysé au sens de talent, c'est-à-dire la capacité non ordinaire d'un individu à faire certaines choses. Il s'agit plutôt de la donation.

${ }^{25}$ Selon Papilloud, C. (2002, p. 150), la loi du don « ... déborde largement le cadre du seul don des choses, qui, pour circuler, en appellent à la dimension principielle du don : que l'autre donne à son tour. »
} 
la solidarité internationale, trois situations se présentent généralement. Primo, le « contre-don » est un discours de gratitude au cours duquel le mot MERCI (ou en langues locales : wolof, sérères) est indéfiniment prononcé. Secundo, le « contre-don » donne lieu à une sorte de démonstration artistico-culturelle des populations bénéficiaires. Il s'agit, pour la plupart du temps, de théâtres relatifs à des thématiques du développement international, de danses traditionnelles locales dont l'imitation des pas par les volontaires internationaux, donne lieu à un mélange de salves d'applaudissements, d'encouragements, de félicitations, de fierté, de démonstrations et de dévoilement de talents latents ou patents, d'hilarité générale ; toutes choses qui revivifient le lien social entre toutes et tous. Tertio, le «contre-don » est matérialisé par une séance de remises de cadeaux, non pas au sens de la ruse (Laurent, 1998), aux volontaires internationaux. Il peut s'agir de divers objets d'art locaux, d'habits locaux et pagnes locaux cousus comme tels ou tout objet traditionnel. Ainsi, pour un même don de la solidarité internationale, ces trois situations de « contre-don » peuvent être distinctes ; tout comme elles peuvent se produire en même, en fonction des circonstances ou du contexte.

\section{Conclusion}

L'initiation à la Solidarité internationale en Afrique subsaharienne présente d'importants enjeux, souvent sous-estimés par la plupart des volontaires internationaux. C'est ce que le présent article, issu d'une expérience de terrain, s'est attelé à identifier. Ainsi, l'analyse a révélé des enjeux de pouvoir, du lien social, des enjeux du don, du contre-don, du don de soi, de temps et la problématique du mimétisme.

Bien que ces points développés n'épuisent pas l'analyse, ils permettent cependant de comprendre des réalités du champ de la Solidarité internationale dans un pays de l'Afrique subsaharienne, qui semblent aller de soi pour les volontaires internationaux. Et il serait louable que la même pratique, c'est-àdire l'initiation au développement international, soit étudiée dans d'autres contextes du Sud pour voir si les enjeux identifiés puis analysés dans le présent article seraient identiques à ceux d'ailleurs.

\section{References:}

1. Arborio, A-M et Fournier, P. 2010. L'enquête et ses méthodes. L'observation directe, Paris : Armand Colin, $3^{\text {ème }}$ édition.

2. Armony, V. 2008. «La société civile et le développement ». In Baudet, P., Schäfer, J. et Haslam, P. (dirs.), Introduction au développement international: approches, acteurs et enjeux (p.197-211). Ottawa : Presses de l'Université d'Ottawa.

3. Athané, F. 2011. Pour une histoire naturelle du don, Paris : Presses Universitaires de France. 
4. Bardin, L. 1993. L’analyse de contenu, Paris : PUF.

5. Beaudet, P. 2009. Qui aide qui ? Une brève histoire de la solidarité internationale au Québec, Montréal : Boréal.

6. Binnet, K. 2013. « Partir comme stagiaire ». In Beaudet, P., Canet, R. et Nguyen, A. (dirs.). Passer de la réflexion à l'action. Les grands enjeux de la coopération et de la solidarité internationale (p.213-2017). Mont-Royal : M éditeur.

7. Boltanski, L. 2007. La souffrance à distance. Morale humanitaire, médias et politique; suivi de La présence des absents, Paris : Gallimard.

8. Bonnafous-Boucher, M. et Dahl Rendtorff, J. 2014. La théorie des parties prenantes. Avant-propos de R. Edward Freeman, Paris : La Découverte. Coll. Repères.

9. Brown, S. 2008. «L'aide publique canadienne à l'Afrique : vers un nouvel âge d'or ?». In Audet, F., Desrosiers, M-E. et Roussel, S. (dirs.), L'aide canadienne au développement (p.267-290). Montréal : Presses Universitaires de Montréal.

10. Caillé, A. 2007. Anthropologie du don. Le tiers paradigme, Paris : La Découverte.

11. Caillé, A. 2012. L’idée même de richesse, Paris : La Découverte.

12. Combessie, J-C. 1996. La méthode en sociologie, Paris : La Découverte. Coll. Repères.

13. Copans, J. 2008. L'enquête et ses méthodes : L'enquête ethnologique de terrain ( $2^{\text {ème }}$ éd.). Paris : Armand Colin.

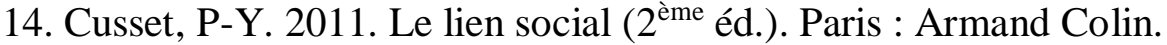

15. Fassin, D. 2010. La raison humanitaire : une histoire morale du temps présent, Paris : Seuil.

16. Godbout, T. J. 2000a. L'Esprit du don, Paris : La Découverte.

17. Godbout, T. J. 2000b. Le don, la dette et l'identité. Homo donator vs homo oeconomicus, Montréal: Boréal.

18. Godelier, M. 2002. L'Énigme du don, Paris : Flammarion.

19. Hénaff, M. 2002. Le prix de la vérité : le don, l'argent, la philosophie, Paris : Seuil.

20. Kaufmann, J-C. 2007. L'enquête et ses méthodes : L'entretien compréhensif ( $2^{\text {ème } e ́ d . ~ R e f o n d u e), ~ P a r i s ~: ~ A r m a n d ~ C o l i n . ~}$

21. Mauss, M. 2007. Essai sur le don : formes et raison de l'échange dans les sociétés archaïques ( $1^{\text {ère }}$ éd.). Paris : Quadrige.

22. Nestor, J. 2016. Un don doit-il être gratuit ? Solidarité et philanthropie, Rennes : Presses Universitaires de Rennes.

23. Noël, G. 2015. Accompagner le développement international. Démarche, instruments et outils, Paris : L'Harmattan. 
24. Oliver de Sardan, J-P. 1995. Anthropologie et développement : Essai en socio- anthropologie du changement social, Paris : Karthala.

25. Papilloud, C. 2002. Le don de relation. Georg Simmel-Marcel Mauss, Paris : L'Harmattan.

26. Laurent, P-J. (1998). Une association de développement au pays mossi. Le don comme ruse, Paris : Karthala.

27. Rist, G. (2013). Le développement. Histoire d'une croyance occidentale ( $4^{\text {ème }}$ éd. revue et augmentée), Paris : Sciences Po.

28. Baudet, P., Schäfer, J. et Haslam, P. (dirs.). (2008). Introduction au développement international : approches, acteurs et enjeux, Ottawa : Presses de l'Université d'Ottawa.

29. Beaudet, P. et Haslam, P.A. (dirs.). (2014). Enjeux et défis du développement international, Ottawa : Presses de l'Université d'Ottawa.

30. Sequera Carvalho, J.A. (2003). Enjeux géopolitiques et nouvelles approches pour la coopération au développement, Paris : L'Harmattan. 